\title{
A reassessment of target-mask interaction in visual backward masking
}

\author{
KATHLEEN CARLSON and MARK S. MAYZNER \\ Loyola University, Chicago, Ilinois 60626
}

\begin{abstract}
Most theories of visual masking concentrate on the effects of a mask on a target. The present study departs from this position and investigates the manner in which a suppressed target alters the perception of a mask. A visual backward masking paradigm was employed, except that subjects were to identify characteristics of the mask rather than the target. A 5 by 5 square matrix of points, composed of either horizontal or vertical vectors, served as the mask. The mask was observed in both the presence and absence of preceding target stimuli. If observed in the absence of a target, the mask points appeared static and simultaneous. If preceded by a target, the mask points appeared to move in accordance with the orientation of the target. This finding implies a mutual sort of target-mask interaction. Several theoretical implications are discussed.
\end{abstract}

"Visual backward masking" refers to a variety of situations in which the recognition of one stimulus (target) is altered by another, spatially overlapping stimulus (mask) which follows it closely in time. In a germinal paper, Turvey (1973) proposed that backward masking usually entails the competition between a target and mask for central decision processors. Presumably, target suppression occurs because target processing is prematurely terminated by a mask.

Like most theories of visual masking, Turvey's (1973) model pays almost exclusive attention to the manner in which a mask alters a target. Thus, a rather one-sided type of target-mask interaction is postulated. Such a model would not expect a mask to be influenced, in any observable way, by a suppressed target. The present paper departs from this position and presents clear evidence that, in at least some stages of visual processing, a suppressed low-energy target can induce discernible changes on a much larger and brighter mask. In our view, this finding has some important theoretical implications.

Our investigation of mask alterations began rather serendipitously during an unpublished backward masking experiment. A computer-based cathode ray tube (CRT) display system was used in that study, and a 5 by 5 square matrix of points served as the mask. If displayed in the absence of a target stimulus, the mask points appeared static and simultaneous. If preceded by a simple vertical target, the mask appeared to consist of vertical vectors moving in a centrifugal fashion toward the left and right sides of the mask perimeter. If the mask was preceded by a horizontal line, it was seen as

This research was supported by Grant BMS75-09800 from the National Science Foundation to the last author. The authors wish to thank Dr. Deborah Holmes and Dr. R. Bowen for their helpful advice and thoughtful assistance during the preparation of this manuscript. a group of horizontal vectors moving centrifugally toward the top and bottom edges of the mask. It should be noted that these target-induced alterations occurred when there was considerable target suppression: Target recognition was only $20 \%$ accurate when the above observations were noted.

The present experiment was conducted to systematically measure the effects of a suppressed target on a visual mask. The paradigm was similar to that used in backward masking studies, except that subjects' attention was focused on the mask rather than on the target. The task was to decide whether the mask was created with horizontal or vertical vectors. A 5 by 5 square matrix of points served as the mask, and it was constructed by displaying either five horizontal or five vertical vectors successively in time. The rate at which the vectors were displayed was well below the threshold of temporal resolution. Thus, if viewed in the absence of a preceding stimulus, the mask points appeared simultaneous.

On the basis of observations described above, three predictions were made. First, when a mask is presented alone, performance would be at a chance level of accuracy (i.e., 50\%). In this condition, it was assumed that subjects would have no movement cues to make their decisions. Second, when a mask is preceded by a stimulus of an orientation identical to that of the mask vectors, performance would be near perfect. This prediction followed from our expectation that the target would induce discernible movement cues that would coincide with the mask's construction. Third, when a mask is preceded by a stimulus of an orientation different from that of the mask vectors, performance would be extremely poor. This last prediction stems from the expectation that mask movement would vary in accordance with the orientation of a preceding stimulus, regardless of the orientation of mask vectors. Thus, in this condition, horizontal masks would appear vertical, and vertical masks would appear horizontal. 


\section{METHOD}

\section{General Design}

Each type of mask (horizontal or vertical) was presented under five different conditions, producing a total of 10 experimental cells. In the first two conditions, each type of mask was observed in the absence of a preceding stimulus. In each of the remaining eight conditions, one of four possible "target" stimuli was displayed a short time before the mask. Each subject was observed for 10 trials in each of the 10 experimental conditions.

\section{Subjects}

Two females and seven males, ranging in age from 22 to 50 years, volunteered to participate in the experiment. All but two of the subjects were graduate students in psychology at Loyola University.

\section{Apparatus}

Stimuli were presented on a VR-14 CRT driven by a PDP-8/E computer. A more detailed description of this general type of system can be found elsewhere (Mayzner, 1968, 1975). The CRT was placed in a darkened room adjacent to the one containing the computer hardware. A small reading lamp was placed behind the CRT so subjects could record their responses on an answer sheet. Subjects viewed the CRT binocularly with the aid of a chinrest positioned approximately $70 \mathrm{~cm}$ from the center of the screen. Each experimental trial was initiated when the subject signaled to the experimenter located in the computer room. The signal was a tone emitted by a walkie-talkie.

\section{Stimuli}

All stimuli consisted of point light sources arranged according to various spatial-temporal parameters. Each point was $.025 \mathrm{~cm}$ in diam and had a display luminance of approximately $1 \mathrm{~mL}$. A 5 by 5 square matrix of points, which served as the mask, was $1.25 \mathrm{~cm}$ long on each side of its perimeter. In the horizontal type of mask, five horizontal vectors (with Vector 1 as the bottom row, Vector 2 the next row, etc.) were successively displayed once every $10 \mathrm{msec}$. Less than $2 \mathrm{msec}$ of off-time separated spatially adjacent vectors. The vertical type of mask was constructed in a similar fashion, except that Vector 1 was the leftmost column of the mask, Vector 2 the next column, etc.

The "target" stimuli presented before the masks consisted of either one or two vectors. Each vector was $1.25 \mathrm{~cm}$ long and was composed of five equally spaced points. The single-vector targets were oriented either horizontally or vertically. The spatial coordinates of these stimuli were identical to those of either the third row (horizontal target) or the third column (vertical target) of the mask. The double-vector targets consisted of two parallel vectors $(.25 \mathrm{~cm}$ separating the vectors) oriented horizontally or vertically. The spatial coordinates of these stimuli overlapped with those of the the second and fourth rows (horizontal target) or the second and fourth columns (vertical target) of the mask.

The duration of masks was 500 msec. For targets, the ontime was kept at a minimum. It took 150 microsec to display single-vector targets, and 300 microsec to display double-vector targets. An interval of $40 \mathrm{msec}$ separated the offset of targets and the onset of masks. This ISI value was chosen because previous work had shown target suppression to be considerable at this value (i.e., recognition was never greater than $25 \%$ ).

\section{Procedure}

Subjects were told that on each trial either a horizontal or vertical type of mask would be presented. The specific nature of the masks was described in detail, but no subject was aware of the experimental predictions. The subjects were instructed to focus their attention on the mask and to ignore any other stimulus which they might perceive prior to the presentation of the mask. Each subject was given an answer sheet on which to record an " $\mathrm{H}$," if he thought the mask was horizontal, or a "V," if it was vertical. They were instructed to guess whenever they were unsure.

Ten practice trials were given before the experiment began. The experimental trials were presented in five blocks of 20 trials each. Each experimental condition was randomly assigned to two positions in each of the five blocks. Each subject was observed in a single experimental session which lasted approximately $20 \mathrm{~min}$.

\section{RESULTS}

The percent number of $\mathrm{H}$ and $\mathrm{V}$ responses given by each subject in each experimental condition was computed. If the target stimuli had no discernible effects on the masks, the distribution of $\mathrm{H}$ and $\mathrm{V}$ responses should have been equal in all conditions. However, Table 1 shows that there were marked differences among the conditions. Moreover, when a target preceded a mask, responses coincided with target orientation on over $90 \%$ of the trials.

To statistically test the experimental hypotheses, the percent number of correct responses in each condition was determined. A response was considered correct if it coincided with the orientation of vectors used to construct a mask. As expected, the mean level of accuracy in the mask-alone condition was $52 \%$. To compare levels of accuracy among experimental conditions, four planned nonorthogonal comparisons were made using Dunn's multiple $t$ test (as described in Kirk, 1968, pp. 79-81). First, conditions of identical targetmask orientation had a significantly greater number of correct responses than the mask-alone condition $(d=.17, p<.05)$. Second, the mask-alone condition had a higher level of accuracy than conditions of different target-mask orientation $(d=.19, p<.05)$. Finally, there was no difference between single-vector and double-vector conditions, either when target-mask orientation was the same $(d=.001, p>.20)$ or different $(\mathrm{d}=.002, \mathrm{p}>.20)$.

It should be noted that subjects reported seeing clear mask movement on most all of the trials. Judging from the data, failure to perceive movement was restricted to conditions with no target. Moreover, when subjects did perceive mask movement, they felt extremely confident of their decisions, even though they were wrong half of the time. In contrast to mask perception, no subject was able to recognize, with any confidence, the exact nature of the target stimuli. Thus, the mask induced considerable target suppression.

Table 1

Mean Percent of $H$ and $V$ Responses

\begin{tabular}{ccccccc}
\hline & \multicolumn{5}{c}{ Target Orientation } \\
\cline { 2 - 4 } $\begin{array}{c}\text { Response } \\
\text { Type }\end{array}$ & None & Single & Double & Single & Double \\
\cline { 3 - 6 } \cline { 5 - 7 } H & .61 & .92 & .93 & .02 & .06 \\
V & .39 & .08 & .07 & .98 & .94 \\
\hline
\end{tabular}




\section{DISCUSSION}

The data are in almost perfect agreement with the preexperimental predictions. A target stimulus, even though suppressed, can induce discernible and systematic changes in a mask. In the present study, mask alterations varied in accordance with target orientation. Such a finding implies that backward masking entails a mutual sort of target-mask interaction, a notion that has not yet been delineated in most interruption models of visual processing (e.g., Turvey, 1973). Clearly, a feasible explanation of the present data must be able to accommodate the masks' mimicry of target orientation, as well as the target suppression observed.

Breitmeyer and Ganz (1976) have recently outlined a model in which backward masking is viewed as the synthesis of a target and a mask in a central store. It is hypothesized that, at certain onset asynchronies, the visual system will combine spatialfrequency components of both a target and a mask into a single, composite iconic representation. In our view, this model implies that some components of a target could be manifested in a composite icon (i.e., mask mimicry), and other components, irretrievably camouflaged therein (i.e., target suppression). Further research is needed to determine the stimulus durations and ISI values at which the mask alterations reported in this study occur. In this way, the efficacy of Breitmeyer's model can be evaluated for various stages of visual processing.

The appearance of movement per se in the masks also needs an explanation. The existence of mask movement in metacontrast, and its relationship to target suppression, have been noted by several investigators (Breitmeyer, Love, \& Wepman, 1974; Kahneman, 1967; Weisstein, Ozog, \& Szoc, 1975). Weisstein and her associates suggested that this movement is mediated by transient cells activated by the target. This explanation, though a feasible one, would have to be modified to fit our data. Transient cells are considered to function as "blob" detectors, signaling the presence of an object without giving information as to its identity. However, the mask movement observed in this experiment bore a systematic relationship with target orientation. Thus, if transient cells were responsible for the mask movement observed in the present study, they were functioning as more than "blob" detectors. They were signaling the orientation, as well as the presence of target stimuli. Further clarification of this problem is needed.

Finally, the present study is relevant to previous reports of differences in recognition thresholds between linear and non- linear inputs (Mayzner, 1972; Uttal, 1975). That finding might have been due, in part, to the manner in which the two types of targets affected the masks used in that research. For example, our subjects reported that, although they could not clearly identify the targets per se, they could have inferred their orientation and geometry on the basis of mask-movement cues. Perhaps nonlinear targets produce less intelligible and less useful mask cues than linear ones. If so, the superiority of linear targets could have been related to the utility of mask cues, rather than to feature extraction per se. We are planning future experiments to investigate this possibility.

\section{REFERENCES}

Breitmeyer, B., \& GANz, L. Implications of sustained and transient channels for theories of visual pattern masking, saccadic suppression, and information processing. Psychological Review, 1976, 83, 1-36.

Breitmeyer, B., Love, R., \& Wepman, B. Contour suppression during stroboscopic motion and metacontrast. Vision Research, 1974, 14, 1451-1456.

KAHNEmAN, D. An onset-onset law for one case of apparent motion and metacontrast. Perception \& Psychophysics, 1967, 2, 577-584.

KIRK, R. Experimental design: Procedures for the behavioral sciences. Belmont, Calif: Brooks/Cole, 1968. Pp. 79-81.

MAYZNER, M. S. The research potential of a computer-based cathode ray tube display system. Behavior Research Methods \& Instrumentation, 1968, 1, 41-43.

MAYZNER, M. S. Visual information processing of alphabetic inputs. Psychonomic Monograph Supplements, 1972, 4, 239-243.

MAYZNER, M. S. Studies in visual information processing in man. In R. Solso (Ed.), Information processing and cognition. Hillsdale, N.J: Lawrence Erlbaum, 1975.

TURVEY, M. T. On peripheral and central processes in vision. Psychological Review, 1973, 80, 1-52.

UtTAL, W. An autocorrelation theory of form detection. Hillsdale, N.J: Lawrence Erlbaum, 1975.

Weisstein, N., Ozog, G., \& Szoc, R. A comparison and elaboration of two models of metacontrast. Psychological Review, 1975, 82, 325-343.

(Received for publication October 26, 1976.) 